\title{
Terapias alternativas e complementares no tratamento de sintomas gastrointestinais em crianças com Transtorno do Espectro Autista
}

\author{
Alternative and complementary therapies in the treatment of gastrointestinal symptoms in \\ children with Autistm Spectrum Disorder
}

\author{
Terapias alternativas y complementarias en el tratamiento de los sintomas \\ gastrointestinales en niños con Trastorno del Espectro Autistico
}

Gilma Sannyelle Silva Rocha ${ }^{1}$, Najra Danny Pereira Lima ${ }^{1}$, Mayanny da Silva Lima ${ }^{1}$, Natália Monteiro Pessoa ${ }^{1}$, Maria Camila da Silva ${ }^{1}$, Valeria Silva Carvalho ${ }^{1}$, Mychelle Maria Santos de

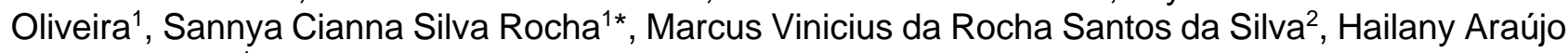
Costa da Silva ${ }^{1}$.

\section{RESUMO}

Objetivo: Buscou-se revisar a literatura cientifica atual sobre a utilização de terapias alternativas para melhora dos sintomas gastrointestinais em crianças com Transtorno do Espectro Autista (TEA). Métodos: Trata-se de uma revisão integrativa da literatura de artigos publicados entre 2009 e 2019, por meio da associação dos descritores: terapias alternativas e complementares, microbiota intestinal e Transtorno do Espectro Autista nas bases de dados Bireme, SciELO e PubMed, Resultados: Foram incluídos nove estudos que demonstraram os benefícios da utilização de terapias alternativas e complementares para melhora de sintomas gastrointestinais em crianças com TEA. As terapias mais utilizadas foram suplementação de vitamina A, prebióticos/ probióticos e enzimas digestivas. A utilização de terapias alternativas e complementares mostra-se como recurso benéfico no tratamento de transtornos gastrointestinais em crianças com TEA. Considerações finais: Poucos estudos com metodologias robustas foram realizados para avaliar o real impacto dessas terapias no tratamento desses sintomas neste público, requerendo mais atenção a esse tema, tendo em vista os seus benefícios comprovados.

Palavras-chave: Terapias alternativas e complementares, Microbiota intestinal, Transtorno do espectro autista.

\begin{abstract}
Objective: We sought to review the current scientific literature on the use of alternative therapies to improve gastrointestinal symptoms in children with Autistic Spectrum Disorder (ASD). Methods: This is an integrative literature review of articles published between 2009 and 2019, through the association of the descriptors: alternative and complementary therapies, intestinal microbiota and Autistic Spectrum Disorder in the databases Bireme, SciELO and PubMed. Results: Nine studies were included that demonstrated the benefits of using alternative and complementary therapies to improve gastrointestinal symptoms in children with ASD. The most used therapies were vitamin A supplementation, prebiotics / probiotics and digestive enzymes. The use of alternative and complementary therapies is shown to be a beneficial resource in the treatment of gastrointestinal disorders in children with ASD. Final considerations: Few studies with robust methodologies have been carried out to assess the real impact of these therapies in the treatment of these symptoms in this audience, requiring more attention to this topic, in view of their proven benefits.
\end{abstract}

Keywords: Alternative and complementary therapies, Intestinal microbiota, Autistic spectrum disorder.

${ }^{1}$ Centro Universitário de Ciências e Tecnologia do Maranhão (UniFacema). Caxias - MA.

2Universidade Federal do Maranhão (UFMA). São Luís - MA.

*E-mail: sannyellerocha17@gmail.com

SUBMETIDO EM: 4/2020

ACEITO EM: 4/2020

PUBLICADO EM: 7/2020 


\section{RESUMEN}

Objetivo: buscamos revisar la literatura científica actual sobre el uso de terapias alternativas para mejorar los síntomas gastrointestinales en niños con trastorno del espectro autista (TEA). Métodos: Esta es una revisión integradora de la literatura de artículos publicados entre 2009 y 2019, a través de la asociación de los descriptores: terapias alternativas y complementarias, microbiota intestinal y trastorno del espectro autista en las bases de datos Bireme, SciELO y PubMed. Resultados: Se incluyeron nueve estudios que demostraron los beneficios del uso de terapias alternativas y complementarias para mejorar los síntomas gastrointestinales en niños con TEA. Las terapias más utilizadas fueron la suplementación con vitamina A, prebióticos / probióticos y enzimas digestivas. Se demuestra que el uso de terapias alternativas y complementarias es un recurso beneficioso en el tratamiento de trastornos gastrointestinales en niños con TEA. Consideraciones finales: pocos estudios con metodologías robustas se han llevado a cabo para evaluar el impacto real de estas terapias en el tratamiento de estos síntomas en esta audiencia, lo que requiere más atención a este tema, en vista de sus beneficios comprobados.

Palabras clave: Terapias alternativas y complementarias, Microbiota intestinal, Trastorno del espectro autista.

\section{INTRODUÇÃO}

O Transtorno do Espectro Autista (TEA) é classificado como um conjunto de desordens que comprometem o desenvolvimento neurológico e com isso proporciona atraso na linguagem, nas habilidades sociais, incluindo a interação social e comunicação não verbal. Até a década de 80 , casos de TEA eram raramente diagnosticados, em que a prevalência era de aproximadamente 5 para cada 10.000 pessoas, todavia, atualmente esses números modificaram e se tornaram cada vez mais comum, algumas estimativas indicam que 1 a cada 54 indivíduos tenham TEA. A incidência do TEA é mais comum no sexo masculino e os primeiros sinais têm sido evidenciado a partir dos 3 anos de idade (AMERICAN PSYCHIATRIC ASSOCIATION, 2014; NEWSCHAFFER CJ, et al., 2007; MAENNER MJ, et al., 2020).

As causas do TEA não estão totalmente elucidadas, mas alguns fatores são apontados como potenciais agentes causais. As principais etiologias propostas, são: genética e ambiental, em que a composição genética é mais aceita entre os pesquisadores. Um estudo mostrou que as alterações epigenéticas causam modificações hereditárias nos cromossomos, a exemplo de alterações na metilação do DNA, que colaboram para o desenvolvimento do TEA. O diagnóstico do TEA é realizado por médicos, em que utilizam para o diagnostico as classificações do Manual Diagnóstico e Estatístico de Transtornos Mentais (DSM-5) (TAYLO MJ, et al., 2020; PENDERGRASS S, et al., 2014).

O TEA apresenta uma condição clinica bastante heterogênea, uma vez que alguns indivíduos possuem comprometimento grave e incapacidade intelectual, enquanto outros indivíduos têm quociente de inteligência acima da média e altos níveis de funcionamento acadêmico e ocupacional. O TEA pode alterar o desenvolvimento infantil, afetando tanto a qualidade quanto a frequência dessas trocas de aprendizado social no início do desenvolvimento (FREEMAN S e KASARI C, 2013).

Além disto, é muito comum pessoas com TEA apresentarem alterações no sistema gastrointestinais. Estima-se que $91 \%$ das crianças com TEA possam desenvolver sintomas gastrointestinais debilitantes, tais como constipação, diarreia, alergia e/ou intolerância alimentar. Esses dados são preocupantes, uma vez que podem comprometer a saúde, porque o desequilíbrio da microbiota intestinal pode proporcionar alterações metabólicas, hormonais, permeabilidade intestinal e outros (BUIE T, et al., 2010). Os principais impactos das modificações no trato gastrointestinal são a perda ou redução da função dos enterócitos, inflamação, dismotilidade, isto pode ocorrer por conta da modificação da composição da microbiota intestinal (PARRACHO HM, et al., 2005; SONG Y, LIU C, FINEGOLD SM, 2004; FRYE RE, et al., 2015).

Considerando isto, a principal forma de tratamento de pessoas com TEA é por meio de fármacos, contudo, ainda possui resultados limitados em decorrência da heterogeneidade individual. Porém, é notório a busca por terapias alternativas e/ou complementares, principalmente por conta das complicações gastrointestinais que podem comprometer o estado nutricional e a saúde geral das pessoas com TEA (MILLWARD C, et al., 
2011). Dessa forma, este estudo teve como objetivo revisar a literatura científica atual sobre a utilização de terapias alternativas para melhora dos sintomas gastrointestinais em crianças com TEA.

\section{MÉTODOS}

O estudo tratou-se de uma pesquisa de revisão integrativa, em que foi desenvolvida obedecendo as seguintes etapas: 1) Definição do tema e pergunta norteadora; 2) Elaboração dos critérios de elegibilidade; 3) Determinação dos dados a serem obtidos, bem como a categorização dos estudos; 4) Análise dos estudos inclusos; 5) Interpretação dos dados dos estudos; 6) Síntese dos resultados (BOTELHO LRR, et al., 2011).

Para nortear este estudo, elaborou-se a seguinte questão de pesquisa: Quais as evidências científicas sobre a utilização de terapias alterativas na melhora de sintomas gastrointestinais em crianças com Transtorno do Espectro Autista?

Para a localização dos estudos que respondessem à pergunta de pesquisa, os descritores foram obtidos a partir dos Descritores em Ciências da Saúde (DeCS): "Terapias alternativas e complementares", "Microbiota gastrointestinal" e "Transtorno do Espectro Autista". A busca foi realizada nas seguintes bases de dados: Literatura Latino-Americana e do Caribe em Ciências da Saúde (LILACS), PubMed e Scientific Eletronic Library Online (SciELO), no período de setembro a outubro de 2019.

Os descritores foram agrupados em cada base de dados utilizando os conectivos na forma booleana AND da seguinte maneira: Transtorno do Espectro Autista AND Terapias alternativas e complementares; Transtorno do Espectro Autista AND Microbiota gastrointestinal; Terapias alternativas e complementares AND Microbiota gastrointestinal. Os seus respectivos descritores em inglês foram obtidos a partir do MeSH: "Alternative and complementary therapies", "Gastrointestinal microbiote", "Autism Spectrum Disorder"

Os critérios de inclusão estabelecidos foram estudos primários sobre a utilizando terapias alternativas em crianças com TEA, revisões sistemáticas e/ou meta-análises, artigos completos disponíveis gratuitamente na integra, artigos na língua portuguesa e inglesa, publicados os últimos 10 anos (2009-2019).

Os critérios de exclusão foram: relatos de experiência, relatórios técnicos, resumos, monografias, artigos disponibilizados apenas mediante taxa de acesso, trabalhos apresentados em eventos científicos, bem como artigos fora da temática deste estudo.

Os estudos foram pré-selecionados segundo os critérios de inclusão e exclusão e de acordo com a estratégia de busca de cada base de dados. Após a identificação dos artigos selecionados no estudo, foi realizada como sequência metodológica: 1. Leitura criteriosa dos títulos obtidos de publicações completas. Onde nessa etapa realizou-se uma leitura prévia do resumo para verificar se estava condizente com o trabalho de pesquisa. 2. Realização de uma leitura dos artigos na integra, sendo observados dados como: nome do autor ou autores, ano da publicação, base de dados, país de origem, delineamento da pesquisa, objetivo do estudo, perfil amostral, intervenção e desfecho.

Para extrair as informações dos artigos selecionados, fez-se uso de um instrumento que permitiu analisar separadamente cada artigo, no nível metodológico quanto aos resultados da pesquisa, permitindo observar suas diferenças, a matriz de síntese. A matriz de síntese tem sido utilizada como ferramenta de extração e organização de dados de revisão da literatura em várias disciplinas, devido à sua capacidade para resumir aspectos complexos do conhecimento e tem por objetivo evitar de erros durante a análise (BOTELHO LRR, et al., 2011).

Foram criadas categorias analíticas que facilitaram a ordenação e a sumarização de cada estudo. Essa categorização foi realizada de forma descritiva, indicando os dados mais relevantes para o estudo.

Para a análise, foram criadas categorizações que facilitaram a ordenação e a sumarização de cada estudo. A categorização foi realizada de forma descritiva, partindo de dados mais simples para o mais complexo. No estudo, foi utilizado a análise em forma estatística e em forma de texto conforme a busca. Por fim, foram utilizados cálculos matemáticos e inferências, assim como figuras, quadros e tabelas produzidas no Programa Excel 2016, para facilitar a visualização e uma melhor compreensão. 


\section{RESULTADOS}

Nove artigos foram incluídos nesta revisão, todos no idioma inglês (100\%). A maioria dos artigos foram publicados nos anos de 2019 (33,3\%), com predomínio de estudos realizados na China (25\%), Egito (22,2\%) e Estados Unidos (22,2\%). Em relação à natureza dos estudos, houve prevalência de ensaio clínicos randomizados $(44,4 \%)$. Em relação às terapias, os objetos de estudo dos artigos foram suplementação de vitamina $A$, suplementação de probióticos e suplementação de enzimas digestivas (Tabela 1).

Os estudos de forma geral apresentaram efeitos positivos em relação ao uso de probioticos, de prebioticos, de enzimas digestivas e da vitamina A sobre o comportamento de crianças com TEA, bem como na modulação da microbiota intestinal, em que repercute em melhora nos sintomas gastrointestinais. Contudo, nota-se que alguns estudos não tiveram efeitos significativos, evidenciando a necessidade de estudos mais específicos e que controle as possíveis variáveis de confusão. 
Tabela 1 - Publicações incluídas segundo autor, ano, tipo de estudo, objetivo, intervenção e conclusão.

\begin{tabular}{|c|c|c|c|c|c|}
\hline Autor/Ano & País & Tipo de Estudo & Objetivo & Intervenção & Conclusão \\
\hline $\begin{array}{l}\text { Munasinghe AS, } \\
\text { et al., } 2010\end{array}$ & Austrália & $\begin{array}{l}\text { Ensaio clínico } \\
\text { randomizado } \\
\text { controlado } \\
\end{array}$ & $\begin{array}{c}\text { Efeito da suplementação de } \\
\text { enzimas digestivas sobre } \\
\text { alterações do TEA }\end{array}$ & $\begin{array}{c}\text { Suplementação com } \\
\text { enzimas digestivas por } 6 \\
\text { meses }\end{array}$ & Não se observou efeitos significativos. \\
\hline $\begin{array}{l}\text { Parracho HM, et } \\
\text { al., } 2010\end{array}$ & Reino Unido & $\begin{array}{l}\text { Ensaio clínico } \\
\text { randomizado }\end{array}$ & $\begin{array}{l}\text { Efeitos de probiótico sobre } \\
\text { comportamento de criança } \\
\text { com TEA }\end{array}$ & $\begin{array}{l}\text { Suplementação com } \\
\text { Lactobacillus plantarum } \\
\text { WCFS1 por } 12 \text { semanas }\end{array}$ & $\begin{array}{c}\text { Modulação da microbiota fecal, melhora } \\
\text { na consistência das fezes e escores } \\
\text { gerais de } \\
\text { comportamento }\end{array}$ \\
\hline $\begin{array}{l}\text { Saad K, et al., } \\
\quad 2015\end{array}$ & Egito & $\begin{array}{c}\text { Ensaio Clínico } \\
\text { randomizado duplo- } \\
\text { cego } \\
\end{array}$ & $\begin{array}{c}\text { Eficácia de enzimas } \\
\text { digestivas em crianças com } \\
\text { TEA }\end{array}$ & $\begin{array}{c}\text { Suplementação de enzimas } \\
\text { digestivas por } 3 \\
\text { Meses }\end{array}$ & $\begin{array}{l}\text { Melhora na impressão geral, sintomas } \\
\text { gastrointestinais, comportamento geral, } \\
\text { resposta emocional }\end{array}$ \\
\hline $\begin{array}{l}\text { Liu Q, et al., } \\
2017\end{array}$ & China & $\begin{array}{l}\text { Estudo piloto duplo- } \\
\text { cego não } \\
\text { randomizado }\end{array}$ & $\begin{array}{c}\text { Papel da } \\
\text { vitamina A nas alterações da } \\
\text { microbiota intestinal e } \\
\text { alterações }\end{array}$ & $\begin{array}{l}\text { Suplementação de vitamina } \\
\text { A por } 6 \text { meses }\end{array}$ & $\begin{array}{c}\text { Redução na proporção de Bacteroidetes/ } \\
\text { Bacteroidales e melhora de } \\
\text { biomarcadores }\end{array}$ \\
\hline $\begin{array}{l}\text { Patusco R e } \\
\text { Ziegler J, } 2018\end{array}$ & $\begin{array}{l}\text { Estados } \\
\text { Unidos }\end{array}$ & Revisão sistemática & $\begin{array}{l}\text { Efeito dos probióticos no } \\
\text { alívio dos sintomas } \\
\text { gastrointestinais em crianças }\end{array}$ & $\begin{array}{l}\text { Suplementação de } \\
\text { probióticos }\end{array}$ & $\begin{array}{l}\text { Melhora na disfunção gastrointestinal, } \\
\text { alteração benéfica da microbiota fecal e } \\
\text { reduzir a gravidade dos sintomas }\end{array}$ \\
\hline $\begin{array}{l}\text { Shaaba YS, } \\
\text { et al., } 2018\end{array}$ & Egito & Estudo prospectivo & $\begin{array}{c}\text { Eficácia e tolerabilidade dos } \\
\text { probióticos em crianças com } \\
\text { TEA }\end{array}$ & $\begin{array}{l}\text { Suplementação de } \\
\text { probioticos por } 3 \text { meses }\end{array}$ & $\begin{array}{c}\text { Efeitos benéficos sobre comportamento e } \\
\text { sintomas gastrointestinais }\end{array}$ \\
\hline $\begin{array}{l}\text { Liu J, et al., } \\
2019\end{array}$ & China & $\begin{array}{l}\text { Revisão sistemática } \\
\text { de ensaios clínicos }\end{array}$ & $\begin{array}{l}\text { Eficácia dos probióticos no } \\
\text { alívio dos sintomas } \\
\text { comportamentais de TEA e } \\
\text { comorbidades gastrointestinal }\end{array}$ & $\begin{array}{l}\text { Suplementação com } \\
\text { probioticos }\end{array}$ & $\begin{array}{c}\text { Melhora na função gastrointestinal após } \\
\text { a suplementação }\end{array}$ \\
\hline $\begin{array}{l}\mathrm{Ng} \text { QX, et al., } \\
2019\end{array}$ & Cingapura & Revisão sistemática & $\begin{array}{c}\text { Efeito de prebioticos e } \\
\text { probioticos sobre sintomas } \\
\text { gastrointestinais }\end{array}$ & $\begin{array}{l}\text { Suplementação com } \\
\text { prebioticos e probioticos }\end{array}$ & $\begin{array}{c}\text { Apesar de alguns efeitos promissores, } \\
\text { existe limitações nos estudos para } \\
\text { garantir a efetividade sobre os sintomas } \\
\text { gastrointestinais }\end{array}$ \\
\hline $\begin{array}{l}\text { Sanctuary MR, } \\
\text { et al., } 2019\end{array}$ & $\begin{array}{l}\text { Estados } \\
\text { Unidos }\end{array}$ & $\begin{array}{l}\text { Ensaio Clínico } \\
\text { Randomizado } \\
\text { Controlado }\end{array}$ & $\begin{array}{c}\text { Efeitos de } \\
\text { probiótico/prebioticos } \\
\text { combinado com colostro } \\
\text { bovino sobre microbioma e } \\
\text { fatores imunes }\end{array}$ & $\begin{array}{l}\text { Suplementação } \\
\text { probiótico/prebiótica por } 12 \\
\text { semanas e suplementação } \\
\text { apenas com prebióticos por } \\
5 \text { semanas. }\end{array}$ & $\begin{array}{l}\text { Redução de sintomas gastrointestinais e } \\
\text { de comportamentos estereotipados }\end{array}$ \\
\hline
\end{tabular}

Fonte: Rocha GSS, et al., 2019. 


\section{DISCUSSÃO}

Os principais problemas gastrointestinais relatados em pessoas com TEA são constipação crônica, diarreia, dor abdominal, fezes com sangue, vômitos e flatulência. Estes problemas são em sua maioria das vezes causados por inflamação do intestino, síndrome do intestino irritável, e dificuldades de digestão. Outra questão que podem colaborar para alterações gástricas assim como o agravamento é a preferência que muitas pessoas com TEA possuem por um único alimento, o que pode também repercutir em uma alteração do estado nutricional a depender do tipo de alimento e da sua composição (GORRINDO P, 2013).

A falta de mastigação adequada é um fator importante nas alterações gástricas, uma vez que podem alterar o processo digestivo dos alimentos, e isto é bastante comum entre crianças. A participação da microbiota intestinal é outro ponto de extrema importância, pois a composição da microbiota pode contribuir para o desequilíbrio intestinal, o que impacta diretamente o sistema imunológicos, contribuindo para desenvolvimento de patologias.

Um fator agravante deste quadro, é o fato de muitas crianças com TEA não consumirem alimentos vegetais, como frutas, verduras e legumes (BUIE T, et al., 2010; WILLIAMS BL, et al., 2012; MCELHANON BO, et al., 2014).

A variação na composição da microbiota intestinal é discutida como uma possível causa de alterações no comportamento do neurodesenvolvimento nessas crianças. Um estudo experimental realizado na Venezuela comprovou uma relação entre graus de disbiose e o neurodesenvolvimento em crianças com TEA (MORENORAMOS AO, et al., 2015).

Levy SE e Hyman SL (2015) relatam que as terapias alternativas e complementares têm sido utilizadas para tratar sintomas de autismo em geral e sintomas comórbidos, como atenção, hiperatividade, irritabilidade, mau humor, sintomas gastrointestinais, convulsões, sono e sensibilidade tátil. Em alguns casos, as famílias selecionam as terapias alternativas quando as terapias convencionais parecem não afetar os sintomas centrais na tentativa de fornecer um tratamento mais abrangente.

Esses tratamentos incluem terapias usadas em combinação com abordagens convencionais (complementares) e, menos comumente, no lugar de tratamentos convencionais (alternativos). Dessa forma, a análise dos estudos resultou em três categorias: Suplementação de vitamina $A$, Suplementação de probióticos/prebióticos e Suplementação de enzimas digestivas.

\section{Suplementação de vitamina A}

A deficiência de vitamina A parece ter uma relação com os sintomas gastrointestinais de crianças autistas. Um estudo realizado na China relatou que as concentrações séricas de vitamina $A$ eram muito menores que as concentrações de ferritina, folato, vitamina B12, $25(\mathrm{OH})$ vitamina $D$ e hemoglobina em crianças com TEA, e sintomas gastrointestinais mais comuns em crianças com TEA do que em crianças com desenvolvimento típico.

Tais resultados mostram que os níveis de vitamina $A$ estavam negativamente correlacionados com escores de sintomas de autismo (LIU X, et al., 2016).

A microbiota intestinal pode favorecer o desenvolvimento e comportamentos alterados por meio da ligação com sistema nervoso neuroendócrino, neuroimune e autonômico.

O fator chave para o impacto destes efeitos será determinado pelo tipo de microrganismo que compõe a microbiota. Uma microbiota saúdavel é composta principalmente por Firmicutes Gram-positivos e os Bacteroidetes Gram-negativos (principalmente Bacteroides, Alistipes, Parabacteroides e Prevotella) (DAVID LA, et al., 2014).

Um ensaio clínico com 64 crianças de 1 a 8 anos de idade com TEA concluíram um estudo de acompanhamento de 6 meses com intervenção da vitamina $A$ evidenciou aumentos significativos na proporção de Bacteroidetes/Bacteroidales e reduções na Bifidobacterium após a suplementação com vitamina A, enquanto não foram observadas alterações significativas nos sintomas do autismo. 
No entanto, os resultados dos autores indicaram que a vitamina A poderia regular a microbiota intestinal e beneficiar parcialmente as crianças com TEA, pois a vitamina $A$ atua na regulação de comunidades microbianas, além disto ela também tem papel chave na regulação do sistema imunológico, impedindo a translocação de microrganismos patógenos pela membrana intestinal (LIU Q, et al., 2017).

Além da melhora nos sintomas gastrointestinais, a suplementação com vitamina $A$ também mostrou eficácia para a melhora dos sintomas do TEA, como evidenciado em um estudo que ofertou vitamina $\mathrm{A}$ a crianças com TEA e observou que elas apresentaram melhora significativa nos sintomas de autismo.

As concentrações séricas de retinol em crianças com TEA aumentaram significativamente e os níveis séricos de 5-HT (5-hidroxitriptamina) diminuíram. Além disso, foram observadas alterações estatisticamente significativas nos níveis de expressão de mRNA de pRAR- $\alpha$ (receptor de ácido retinoíco alfa), pRAR- $y$ (receptor de ácido retinoíco gama) e TpH-1 (triptofano hidroxilase 1) após a suplementação em comparação à linha de base (GUO M, et al., 2018).

\section{Suplementação de probióticos/prebióticos}

O mecanismo exato pelo qual os probióticos exercem potenciais efeitos terapêuticos não é conhecido, mas existem várias hipóteses. A resposta mais direta é que pacientes com TEA possuem microbiota intestinal significativamente alterada; é essa microbiota intestinal alterada que origina os problemas gastrointestinais.

Os probióticos presumivelmente reduzem a inflamação intestinal através de uma variedade de mecanismos, como a redução permeabilidade da barreira intestinal, regulação negativa de citocinas inflamatórias e outros efeitos imunomoduladores, o que, por sua vez, ajudam a restaurar a microbiota intestinal de volta aos níveis normais, melhorando os sintomas gastrointestinais concomitantes (NG XQ, et al., 2019).

Níveis elevados de inflamação da mucosa têm sido associados a vários distúrbios gastrointestinais, incluindo síndrome do intestino irritável e doença inflamatória intestinal (NG XQ, et al., 2018; LI Q, et al., 2017). Além disso, foi reportado que as alterações da microbiota bacteriana em indivíduos com TEA resultam na expansão de Candida, a qual pode liberar amônia e toxinas que podem induzir comportamentos autistas e pioraria a disbiose (LI Q, et al., 2017).

Um estudo piloto randomizado, duplo-cego e controlado realizado nos Estados Unidos mostrou que uma suplementação combinada de 12 semanas de probióticos- prebióticos (Bifidobacterium longum subsp infantis + colostro bovino) vs 5 semanas de suplementação apenas com prebióticos (colostro bovino) em crianças de 2 a 11 anos com comorbidades de TEA e distúrbios gastrointestinais mostrou que a combinação de suplementos foi bem tolerada pelas crianças, houve uma redução na frequência de certos sintomas gastrointestinais, tais como dor ao evacuar, frequência de diarreia e consistência das fezes. Os autores explicam que essa melhoria pode ser explicada por uma redução na produção de IL-13 (interleucina 13) e TNF- $\alpha$ (fator de necrose tumoral alfa) em alguns participantes (SANCTUARY MR, et al., 2019).

O aumento de marcadores inflamatórios, tais como interleucina 1, 6, 13, e TNF- a estão associados a alterações intestinais importantes que geram impactos agressivos no sistema gastrointestinal de pessoas com TEA tendo como consequência alterações comportamentais. Neste sentido a diminuição destes marcadores melhoram a função gastrointestinal e alivia sintomas comportamentais e gastrointestinais (MOLLOY CA, et al., 2006).

Da mesma forma, após 3 meses de suplementação com probióticos (cada grama contém $100 \times 106$ unidades formadoras de colônias de três linhagens probióticas; Lactobacillus acidophilus, Lactobacillus rhamnosus e Bifidobacteria longum), Shaaban YS, et al. (2018) descobriram que a PCR de fezes de crianças autistas mostrou aumentos nas contagens de colônias dos níveis de Bifidobacteria e Lactobacilli, com melhorias significativas na gravidade do autismo e sintomas gastrointestinais.

No estudo conduzido por Parracho HM, et al. (2010), foi demonstrado que a administração oral diária de 4,5 $\times 1010$ UFC de L. plantarum WCFS1 uma vez ao dia por 3 semanas em 17 crianças com TEA aumentou significativamente o número de lactobacilos e enterococos na microbiota fecal em comparação com o placebo. 
A alimentação com probióticos também levou a uma contagem de Clostridium coccoides rectale significativamente menor do que o placebo.

\section{Suplementação de enzimas digestivas}

A utilização de suplementação com enzimas digestivas é baseada na capacidade destas de remover ou digerir substancias que necessitam de um esforço maior do sistema digestório. Considerando que algumas crianças com TEA possuem alergia a caseira ou glúten, o uso de enzimas seria um possível alvo terapêutico (PARMEGGIANI A, et al., 2014).

Um ensaio clínico randomizado controlado desenvolvido na Austrália mostrou que a suplementação de enzimas digestivas por 6 meses não teve efeito significativo na melhora dos sintomas de autismo; no entanto, possíveis efeitos na melhoria da variedade de alimentos justificam uma investigação mais detalhada (MUNASINGHE SA, et al., 2010).

Todavia outro estudo apresentou um desfecho diferente, uma vez que a suplementação por 3 meses com enzimas digestivas teve efeito significativo sobre a resposta emocional, escore de TEA, de impressão geral, comportamento geral e sintomas gastrointestinais (SAAD K, et al., 2015). A diferença entre estudos é o tempo de suplementação, é notório que o menor tempo teve efeitos significativos.

\section{Questões relevantes}

Foi evidenciado nesta revisão diversos desfechos associados as terapias complementares em pessoas com TEA. A suplementação de vitamina A, prebioticos/probioticos, enzimas digestivas, apresentam efeitos distintos em relação ao sistema gastrointestinal e por conseguinte no comportamento de pessoas com TEA. Alguns apresentam efeitos benéficos, todavia é importante considerar a saúde da pessoa de forma geral (WHITELEY P, et al., 2010).

Os distúrbios gastrointestinais no TEA são causados também por um padrão alimentar diferente. As consequências mais avanças destes distúrbios são refluxo gastroesofágico, gastroenterite e alergias alimentares.

As estimativas sugerem que $95 \%$ da constipação infantil pode ser funcional, sem uma causa fisiológica subjacente, e muitas crianças com TEA apresentam problemas de higiene não-orgânicos que podem precipitar ou desempenhar um papel no desenvolvimento da constipação, incluindo a aquisição ausente ou tardia do treinamento do intestino e maiores taxas de comportamentos problemáticos relacionados a mudanças na rotina de higiene (D'CRUZ AM, et al., 2013). A retenção fecal no TEA também pode ocorrer devido a dificuldades com estímulos sensoriais, processamento sensorial e problemas motores, levando à alteração da motilidade gastrointestinal e fisiologia da defecação.

Também é possível que taxas elevadas de constipação estejam relacionadas à onipresença de seletividade alimentar nessa população, pois os padrões alimentares frequentemente associados ao TEA envolvem alta ingestão de alimentos processados e carecem de frutas e vegetais que contenham fibras, 0 que fornece um laxante natural efetuar e diminuir o tempo de trânsito intestinal (D'CRUZ AM, et al., 2013).

\section{CONSIDERAÇÕES FINAIS}

Esta revisão forneceu evidências sugestivas sobre o potencial benefícios das terapias alternativas e complementares na melhoria de sintomas gastrointestinais em crianças com TEA e consequentemente em parâmetros comportamentais. No entanto, há pouca disponibilidade de pesquisas robustas sobre intervenção dessas terapias para TEA disponíveis na literatura, e as pessoas de modo geral possuem amostras pequenas, o que limita ainda mais a qualidade dessa revisão e consequentemente a possibilidade de uma conclusão mais efetiva.

Outro ponto a ser destacado foi a identificação de apenas um estudo utilizando suplementação de vitamina A e apenas dois estudos utilizando enzimas digestivas, sendo o último publicado há quatro anos, para o tratamento desses sintomas. A maioria dos estudos analisados teve como foco a suplementação de 
probióticos/prebióticos, o que inviabiliza a comparação da eficácia entre as terapias. Nesse contexto, sugerese a realização de estudos com metodologias robustas utilizando terapias diferentes no intuito de verificar o seu real potencial na melhora dos sintomas gastrointestinais e, consequentemente, sintomas comportamentais do TEA.

\section{REFERÊNCIAS}

1. AMERICAN PSYCHIATRIC ASSOCIATION (APA). Manual diagnóstico e estatístico de transtornos mentais: DSM-5. Associação Brasileira de Psiquiatria. Porto Alegre: Artmed, 2014.

2. BOTELHO LLR, et al. O método da revisão integrativa nos estudos organizacionais. Gestao Soc, 2011, 5(11):121136.

3. BUIE T, et al. Evaluation, diagnosis, and treatment of gastrointestinal disorders in individuals with ASDs: a consensus report. Pediatrics, 2010, 125 (Supl.1), S1-S18.

4. DAVID LA, et al. Diet rapidly and reproducibly alters the human gut microbiome. Nature. 2014;505(7484):559-563. doi:10.1038/nature12820.

5. D'CRUZ AM, et al. Reduced behavioral flexibility in autism spectrum disorders. Neuropsychology. 2013;27(2):152160.

6. FREEMAN S e KASARI C. Parent-child interactions in autism: Characteristics of play. Autism, 2013, 17(2), 147-161.

7. FRYE RE, et al. Gastrointestinal dysfunction in autism spectrum disorder: the role of the mitochondria and the enteric microbiome. Microb Ecol Health Dis, Estados Unidos, 2015, 26(1), 27458.

8. GORRINDO P. Gastrointestinal dysfunction in Autism. Autism Res, 2013, 5(2):101-08.

9. GUO M, et al. Vitamin A improves the symptoms of autism spectrum disorders and decreases 5-hydroxytryptamine (5-HT): A pilot study. Brain Res Bull, Estados Unidos, 2018, 137: 35-40.

10. LEVY SE e HYMAN SL. Complementary and alternative medicine treatments for children with autism spectrum disorders. Child Adolesc Psychiatr Clin N Am. 2015;24(1):117-143.

11. LIU X, et al. Correlation between nutrition and symptoms: nutritional survey of children with autism spectrum disorder in Chongqing, China. Nutrients, Suiça, 2016, 8(5): 294.

12. LIU Q, et al. The gut microbiota and Autism Spectrum Disorders. Front Cell Neurosci, Suiça, 2017, 11: 120.

13. LIU J, et al. Probiotic therapy for treating behavioral and gastrointestinal symptoms in Autism Spectrum Disorder: A systematic review of clinical trials. Curr Med Sci, China, 2019, 39; (2): 173-184.

14. MAENNER MJ, et al. Prevalence of Autism Spectrum Disorder Among Children Aged 8 Years - Autism and Developmental Disabilities Monitoring Network, 11 Sites, United States, 2016. MMWR Surveill Summ 2020;69(No. SS-4):1-12.

15. McELHANON BO, et al. Gastrointestinal symptoms in Autism Spectrum Disorder: A meta-analysis abstract. Pediatrics, 2014, 133(5):872-83.

16. MILLWARD C, et al. Gluten and casein-free diets for autistic spectrum disorder. Cochrane Database Syst Rev, 2008, 16:CD003498.

17. MORENO-RAMOS OA, et al. Whole-Exome Sequencing in a South American Cohort Links ALDH1A3, FOXN1 and Retinoic Acid Regulation Pathways to Autism Spectrum Disorders. PLoS One. 2015;10(9):e0135927.

18. MOLLOY CA, et al. Elevated cytokine levels in children with autism spectrum disorder. Journal of neuroimmunology. 2006;172(1-2):198-205. pmid:16360218.

19. MUNASINGHE SA, et al. Digestive enzyme supplementation for Autism Spectrum Disorders: A double-blind randomized controlled trial. J Autism Dev Disord, Estados Unidos, 2010, 40(9): 1131-1138.

20. NEWSCHAFFER CJ, et al. The epidemiology of autism spectrum disorders. Annual Review of Public Health, 2007, 28: 235-258.

21. NG QX, et al. A systematic review of the role of prebiotics and probiotics in Autism Spectrum Disorders. Medicina (Kaunas), 2019, 55; (5): 129.

22. PARMEGGIANI A. Gastrointestinal disorders and autism. In: Patel VB, Preedy VR, Martin CR, editors. Comprehensive guide to autism. New York: Springer Science plus Business Media; 2014. pp. 2035-2046.

23. PARRACHO HM, et al. A double-blind, placebo-controlled, crossover-designed probiotic feeding study in children diagnosed with autistic spectrum disorders. Int J Probiotics Prebiotics, Estados Unidos, 2010, 5 (2): 69-74.

24. PATUSCO R e ZIEGLER J. Role of probiotics in managing gastrointestinal dysfunction in children with Autism Spectrum Disorder: An update for practitioners. Adv Nutr, Estados Unidos,2018, 9 ( 5): 637-650.

25. PENDERGRASS S, GIRIRAJAN S, SELLECK S. Uncovering the etiology of autism spectrum disorders: genomics, bioinformatics, environment, data collection and exploration, and future possibilities. Pacific Symposium on Biocomputing. Pacific Symposium on Biocomputing, 2014, 422-426. 
26. SAAD K, et al. A randomized, placebo-controlled trial of digestive enzymes in children with Autism Spectrum Disorders. Clin Psychopharmacol Neurosci, Coreia do Sul, 2015, 13 (2): 188-193.

27. SHAABAN YS, et al. O papel dos probióticos em crianças com autismo espectro transtorno: Um estudo prospectivo, aberto, Nutritional Neuroscience, 2018, 21: 9, 676-681,

28. SANCTUARY MR, et al. Pilot study of probiotic/colostrum supplementation on gut function in children with autism and gastrointestinal symptoms. PLoS One, Estados Unidos, 2019, 14 (1): e0210064.

29. SONG Y, LIU C, FINEGOLD SM. Real-time PCR quantitation of clostridia in feces of autistic children. Appl Environ Microbiol, 2004, 70(11):6459-6465.

30. TAYLOR MJ, et al. Etiology of Autism Spectrum Disorders and Autistic Traits Over Time. JAMA Psychiatry, $2020,6$.

31. WILLIAMS BL, et al. Application of novel PCR-based methods for detection, quantitation, and phylogenetic characterization of Sutterella Species in intestinal biopsy samples from Children with Autism and gastrointestinal disturbances. MBio, 2012, 3(1):1-11.

32. WHITELEY P, et al. The ScanBrit randomised, controlled, single-blind study of a gluten- and casein-free dietary intervention for children with autism spectrum disorders. Nutr Neurosci. 2010; 13:87-100. 